\title{
Quality of Evidence Supporting the Role of Acupuncture for the Treatment of Irritable Bowel Syndrome
}

\author{
Jinke Huang $\mathbb{D},^{1}$ Mengxiong Lu $\left(\mathbb{D},{ }^{2}\right.$ Yijun Zheng $\mathbb{D}^{1},{ }^{1}$ Jinxin $M a\left(\mathbb{D},{ }^{2}\right.$ Xiangxue Ma $\left(\mathbb{D},{ }^{1}\right.$ \\ Yitian Wang $\mathbb{D}$, ${ }^{1}$ Kunli Zhang $\mathbb{D}{ }^{1}$ Fengyun Wang $\mathbb{D}$, ${ }^{1,2}$ and Xudong Tang $\mathbb{D}^{1,2,3}$ \\ ${ }^{1}$ Department of Gastroenterology, Xiyuan Hospital of China Academy of Chinese Medical Sciences, Beijing, China \\ ${ }^{2}$ Department of Gastroenterology, Peking University Traditional Chinese Medicine Clinical Medical School (Xiyuan), \\ Beijing, China \\ ${ }^{3}$ China Academy of Chinese Medical Sciences, Beijing, China
}

Correspondence should be addressed to Fengyun Wang; wfy811@163.com and Xudong Tang; txdly@sina.com

Received 8 October 2021; Revised 1 December 2021; Accepted 2 December 2021; Published 15 December 2021

Academic Editor: Noureddin Nakhostin Ansari

Copyright (C 2021 Jinke Huang et al. This is an open access article distributed under the Creative Commons Attribution License, which permits unrestricted use, distribution, and reproduction in any medium, provided the original work is properly cited.

Objectives. To systematically collate, appraise, and synthesize the current evidence on acupuncture for irritable bowel syndrome (IBS). Methods. Systematic reviews (SRs)/meta-analyses (MAs) of acupuncture for IBS were searched in eight databases. For quality evaluation of the enrolled studies, Assessment of Multiple Systematic Reviews 2 (AMSTAR-2) was used for methodological quality, Preferred Reporting Item for Systematic Reviews and Meta-Analyses (PRISMA) for reporting quality, and Grading of Recommendations Assessment, Development, and Evaluation (GRADE) for evidence quality. Results. Ten studies were included in our review. According to AMSTAR-2, only one study met all the criteria and was rated as high methodological quality, and the rest were rated as low or very low methodological quality. According to the PRISMA checklist, most of the items were fully reported, with the exception of Q5 (protocol and registration), Q8 (search), and Q27 (funding). With the GRADE system, no outcome measure was rated as high quality. Conclusions. Acupuncture may be a promising therapy for IBS. However, this conclusion must be treated with caution since the quality of SRs/MAs providing evidence is generally low.

\section{Introduction}

Irritable bowel syndrome (IBS) is a functional gastrointestinal disorder characterized by recurrent abdominal pain accompanied by abnormal or altered defecation habits [1]. From country to country, the prevalence of IBS ranges from $1.1 \%$ to $45.0 \%$ [2], with global estimates of $11.2 \%$ [3] in Rome, 5.9\% in China [4], and 7.1\% in the United States [3]. This disorder not only has a marked negative impact on quality of life (QOL) and work productivity but also increases medical healthcare costs and imposes a huge socioeconomic burden $[5,6]$. It is reported that the annual direct cost per patient due to IBS is estimated to be $\$ 348$ to $\$ 8,750$ and the indirect cost is $\$ 355$ to $\$ 3,344$ [7].

The pathophysiology of IBS is poorly understood and is currently thought to represent a complex interplay among the gut microbiota, mucosal immune system, impaired mucosal barrier function, visceral hypersensitivity, gut motility, and alterations in the gut-brain axis [8-10]. The conventional medication (CM) recommended to alleviate the symptoms include antispasmodics, fiber supplementation antidepressants, and probiotics $[2,8]$. However, the effects are limited and accompanied by various side effects [11]. As a nonpharmacological treatment technique, acupuncture is believed to be beneficial to IBS based on the theory of the visceral hyperalgesia theory of the central nervous system.

Acupuncture is becoming more widely used, and the number of published systematic reviews (SRs) and meta-analyses (MAs) has increased, but the evidence they provide for acupuncture for IBS is not always consistent. SR/MA is considered the gold standard for assessing the effectiveness of clinical interventions; however, high-quality SRs/MAs can provide reliable evidence, while low-quality SRs/MAs might 
instead mislead clinical decision-making [12]. Thus, there may be a gap between evidence-based clinical implementation of acupuncture and its actual implementation in real-world dynamics. Clinical decision-making requires a comprehensive overview of the available evidence in order to identify potential benefits and harms of the intervention [13]. Within this framework, the overview of SRs/MAs is a relatively new approach, which aims to summarize and evaluate the strength of the evidence provided in multiple SRs/MAs [14]. By mapping the evidence in the real-world implementation field of acupuncture, an umbrella review will help draw a clear link between the need to address uncertainty and advancing clinical knowledge a priori [15]. Therefore, we conducted this study.

\section{Methods}

The Cochrane criteria and the statements of Preferred Reporting Item for Systematic Reviews and Meta-Analyses (PRISMA) [16] were followed to carry out this overview. The protocol was registered in PROSPERO (CRD42021228185).

2.1. Strategy for Search. PubMed, Cochrane Library, Web of Science, Embase, Chinese Scientific Journal Database, CNKI, VIP, and Wanfang were systematically searched from inception to July, 2021. Irritable bowel syndrome, acupuncture, systematic review, and meta-analyses were used as search key terms. A search strategy used for PubMed is shown in Table 1.

2.2. Criteria Used to Consider Studies. The studies that met the following criteria were included: (1) SRs/MAs based on randomized controlled trials; (2) the Rome I-IV criteria were adopted as diagnostic criteria for IBS; (3) the experimental intervention was acupuncture or a combination of acupuncture plus medications and the control intervention was Sham acupuncture or CM; and (4) outcome measures should be effective rate, recurrence rate, IBS symptom scores, IBS-QOL, and Symptom Severity Scale of IBS (IBSSSS). The studies that met the following criteria were excluded: (1) duplicate publications; (2) updated SRs/MAs; (3) dissertations without peer review; and (4) the control intervention that included acupuncture.

2.3. Literature Selection and Data Extraction. Literature selection and data extraction were carried out by two independent authors, respectively. For literature selection, titles and abstracts were first screened and then, the full text of potentially relevant studies was further reviewed to determine eligibility. In addition to the outcomes of metaanalyses, data regarding the characteristics of the studies and subjects, details of the treatments, and methods of the SRs/ MAs were extracted. Any discrepancies were resolved by discussion.

2.4. Quality Assessment. Quality assessment was carried out by two independent authors. The Assessment of Multiple Systematic Reviews 2 (AMSTAR-2) [17], PRISMA tool, and
Grading of Recommendations Assessment, Development, and Evaluation (GRADE) [18] were used to evaluate the methodological quality, reporting quality, and evidence quality, respectively. Any discrepancies were resolved by discussion.

\section{Results}

3.1. Included Studies. As shown in Figure 1, the literature search identified 243 citations, and after removing the duplicates, 173 citations were further eliminated, 167 of which were excluded. Finally, 10 studies [19-28] met the inclusion criteria.

3.2. Study Characteristics. As shown in Table 2, 10 MAs published from 2010 to 2020 were enrolled in this overview. Half of these studies were published in English, with the number of trails ranging from 6 to 41 and the subjects ranging from 664 to 3440 . The experimental intervention was mainly acupuncture or a combination of acupuncture plus medications, and the control intervention was mainly Sham acupuncture or CM characteristics.

\subsection{Quality Assessment}

3.3.1. Methodological Appraisal. According to AMSTAR-2, only one review met all items and was rated as high methodological quality, and the rest were rated as low or critically low methodological quality. Key items affecting the methodological quality were item 2 (established protocol), item 4 (comprehensive search strategy), and item 7 (a list of excluded trails). Further details are shown in Figures 2 and 3.

\subsubsection{Reporting Quality Appraisal. According to PRISMA} checklists, most of the items were fully reported in these included reviews, with the exception of Q5 (protocol and registration), Q8 (search), and Q27 (funding). Further details are given in Table 3.

3.3.3. Evidence Quality Classification. 25 outcome indicators regarding the effects of acupuncture for IBS were extracted from the included studies. With GRADE, 12 outcome indicators were rated as moderate quality and the rest were rated as low or critically low quality. The risk of bias, imprecision, inconsistency, and publication bias were the main reasons for evidence degradation (Table 4).

\subsection{Description of Efficacy}

3.4.1. Effect of the Interventions. Relative effects of the outcome indicators regarding the effectiveness of acupuncture for IBS are shown in Table 4. Two studies [20, 23] compared the effects of acupuncture and Sham acupuncture, and reportedly no statistically significant difference was found in effective rate, IBS-QOL, or IBS-SSS. Nine studies $[19,20,22-28]$ compared the effects of acupuncture and CM, and results revealed that patients receiving acupuncture 
TABle 1: Search strategy for the PubMed database.

\begin{tabular}{|c|c|}
\hline Query & Search term \\
\hline \#1 & Irritable bowel syndrome [Mesh] \\
\hline$\# 2$ & $\begin{array}{c}\text { Irritable bowel syndrome [Title/abstract] OR irritable colon syndrome [Title/abstract] OR irritable colon [Title/abstract] OR } \\
\text { gastrointestinal syndrome [Title/abstract] OR colon spasm [Title/abstract] OR allergic colitis [Title/abstract] OR colon allergy } \\
\text { [Title/abstract] OR IBS [Title/abstract] }\end{array}$ \\
\hline \#3 & $\# 1$ OR \#2 \\
\hline \#4 & Acupunct \\
\hline$\# 5$ & $\begin{array}{c}\text { Acupuncture [title/abstract] OR pharmacoacupuncture [title/abstract] OR acupotomy [title/abstract] OR acupotomies [title/ } \\
\text { abstract] OR pharmacopuncture [title/abstract] OR needle [title/abstract] OR needling [title/abstract] OR dry-needling [title/ } \\
\text { abstract] OR body-acupuncture [title/abstract] OR electroacupuncture [title/abstract] OR electro-acupuncture [title/abstract] OR } \\
\text { auricular acupuncture [title/abstract] OR warm needle [title/abstract] }\end{array}$ \\
\hline \#6 & (5) \\
\hline \#7 & eta-analysis \\
\hline$\# 8$ & Systematic review [title/abstract] OR meta-analysis [title/abstract] OR meta-analyses [title/abstract] \\
\hline \#9 & $\# 7$ OR \#8 \\
\hline$\# 10$ & \#3 AND \#6 AND \#9 \\
\hline
\end{tabular}
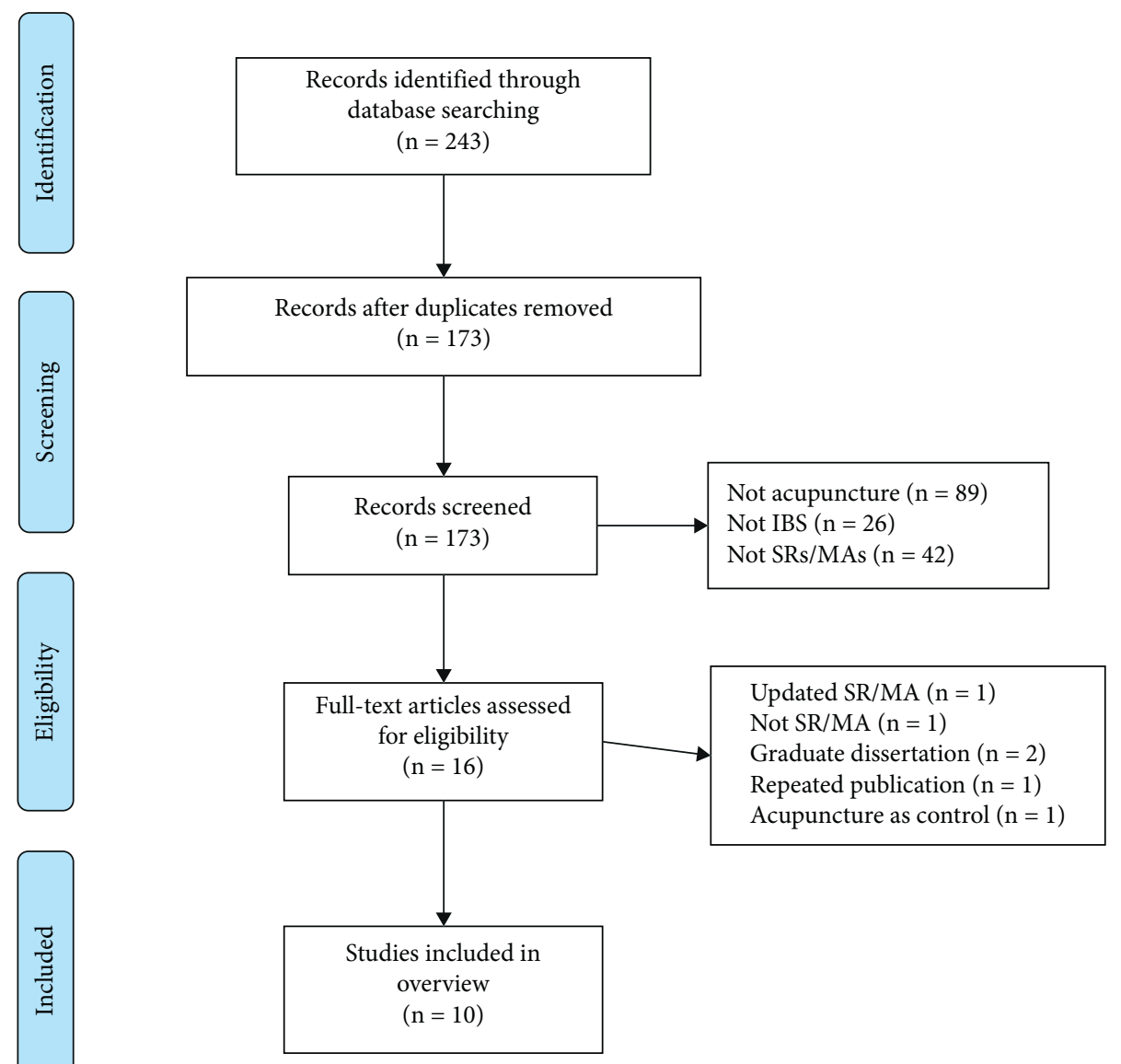

FIgURE 1: Literature screening flow chart.

therapy showed a greater improvement in effective rate, recurrence rate, weekly defecation, IBS symptom scores, IBS-QOL, and IBS-SSS than patients receiving CM. One study [21] compared the effects of acupuncture plus Chinese herbal medicine and $\mathrm{CM}$, and results revealed that patients receiving combination therapy reported a significantly greater improvement in effective rate and abdominal pain than patients receiving CM. 
TABLE 2: Baseline characteristics of included reviews.

\begin{tabular}{|c|c|c|c|c|c|c|c|}
\hline Studies & Country & $\begin{array}{c}\text { Trials } \\
\text { (subjects) }\end{array}$ & $\begin{array}{l}\text { Experimental } \\
\text { Intervention }\end{array}$ & $\begin{array}{c}\text { Control } \\
\text { Intervention }\end{array}$ & $\begin{array}{c}\text { Quality } \\
\text { assessment }\end{array}$ & $\begin{array}{c}\text { Meta- } \\
\text { analyses }\end{array}$ & Results summary \\
\hline Guo et al. [19] & China & $31(3234)$ & AT & $\mathrm{CM}$ & $\begin{array}{l}\text { Cochrane } \\
\text { criteria }\end{array}$ & Yes & $\begin{array}{l}\text { Acupuncture was an effective and safe } \\
\text { therapy for IBS. }\end{array}$ \\
\hline $\begin{array}{l}\text { Zheng et al. } \\
{[20]}\end{array}$ & China & $41(3440)$ & $\mathrm{AT}, \mathrm{AT}+\mathrm{CM}$ & $\begin{array}{l}\text { Sham AT, } \\
\text { CM }\end{array}$ & $\begin{array}{l}\text { Cochrane } \\
\text { criteria }\end{array}$ & Yes & $\begin{array}{l}\text { The effect of acupuncture on IBS was } \\
\text { better than that of CM, which could } \\
\text { be used as an adjuvant therapy in } \\
\text { clinical practice. }\end{array}$ \\
\hline Yan et al. [21] & China & $21(1834)$ & $\mathrm{AT}+\mathrm{CHM}$ & $\mathrm{CM} ; \mathrm{CHM}$ & $\begin{array}{l}\text { Cochrane } \\
\text { criteria }\end{array}$ & Yes & $\begin{array}{l}\text { The combination of acupuncture and } \\
\text { Chinese herbal medicine was effective } \\
\text { and safe in the treatment of IBS. }\end{array}$ \\
\hline $\begin{array}{l}\text { Chao and } \\
\text { Zhang [22] }\end{array}$ & China & $6(664)$ & AT & $\begin{array}{l}\text { Sham AT, } \\
\text { CM }\end{array}$ & Jadad & Yes & $\begin{array}{l}\text { Acupuncture was significant in } \\
\text { relieving the symptoms of IBS. }\end{array}$ \\
\hline $\begin{array}{l}\text { Manheimer } \\
\text { et al. [23] }\end{array}$ & $\begin{array}{l}\text { United } \\
\text { States }\end{array}$ & $17(1806)$ & AT & $\begin{array}{l}\text { Sham AT, } \\
\text { CM }\end{array}$ & $\begin{array}{l}\text { Cochrane } \\
\text { criteria }\end{array}$ & Yes & $\begin{array}{l}\text { The effect of acupuncture on IBS was } \\
\text { better than that of CM, which could } \\
\text { be used as an adjuvant therapy in } \\
\text { clinical practice. }\end{array}$ \\
\hline $\begin{array}{l}\text { Fu and Jiang } \\
{[24]}\end{array}$ & China & $23(1685)$ & AT & $\begin{array}{c}\mathrm{CM} \\
\mathrm{AT}+\mathrm{CM}\end{array}$ & Jadad & Yes & $\begin{array}{c}\text { Acupuncture therapy was superior to } \\
\text { conventional CM in the treatment of } \\
\text { IBS. }\end{array}$ \\
\hline $\begin{array}{l}\text { Deng et al. } \\
\text { [25] }\end{array}$ & China & $17(1333)$ & $\mathrm{AT} ; \mathrm{AT}+\mathrm{CM}$ & $\begin{array}{l}\text { CM; Sham } \\
\mathrm{AT}+\mathrm{CM}\end{array}$ & Jadad & Yes & $\begin{array}{l}\text { Acupuncture for IBS was superior to } \\
\text { conventional treatment, which could } \\
\text { improve the clinical symptoms and } \\
\text { reduce the recurrence rate of patients. }\end{array}$ \\
\hline Li et al. [26] & China & $12(715)$ & $\mathrm{AT}$ & $\mathrm{CM}$ & $\begin{array}{l}\text { Cochrane } \\
\text { criteria }\end{array}$ & Yes & $\begin{array}{l}\text { The evidence of this study was not } \\
\text { sufficient to prove that the efficacy of } \\
\text { acupuncture was better than CM. }\end{array}$ \\
\hline Pei et al. [27] & China & $11(969)$ & $\mathrm{AT} ; \mathrm{AT}+\mathrm{CM}$ & $\begin{array}{l}\text { CM; Sham } \\
\text { AT + CM }\end{array}$ & $\begin{array}{l}\text { Cochrane } \\
\text { criteria }\end{array}$ & Yes & $\begin{array}{l}\text { Acupuncture for IBS was better than } \\
\text { the CM treatment. }\end{array}$ \\
\hline $\begin{array}{l}\text { Zhao et al. } \\
{[28]}\end{array}$ & China & $10(810)$ & AT & $\mathrm{CM}$ & Jadad & Yes & $\begin{array}{l}\text { The effect of acupuncture on IBS was } \\
\text { superior to that of western medicine. }\end{array}$ \\
\hline
\end{tabular}

AT: acupuncture therapy; CHM: Chinese herbal medicine.

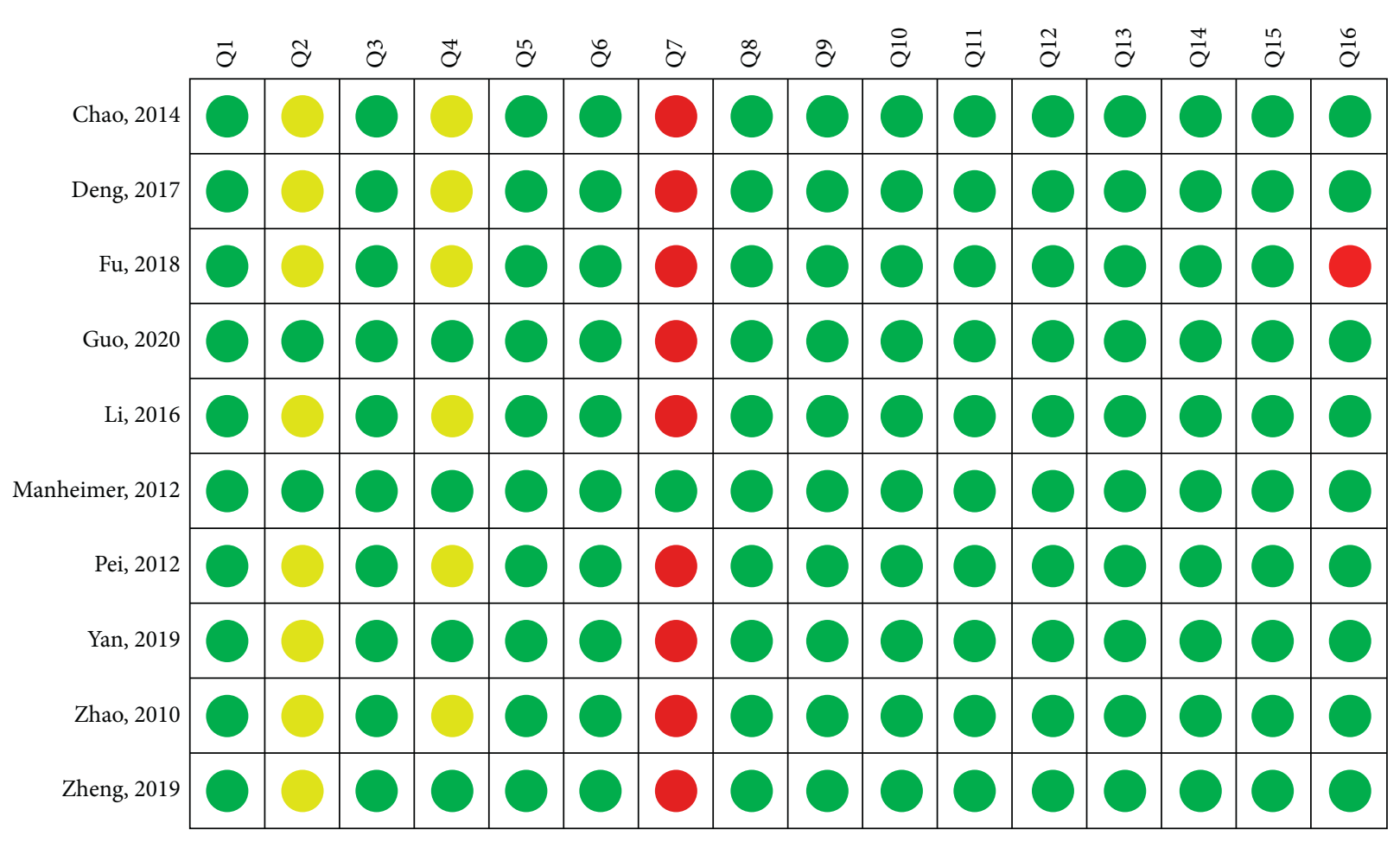

FIgURE 2: Summary of the AMSTAR-2 assessments. 


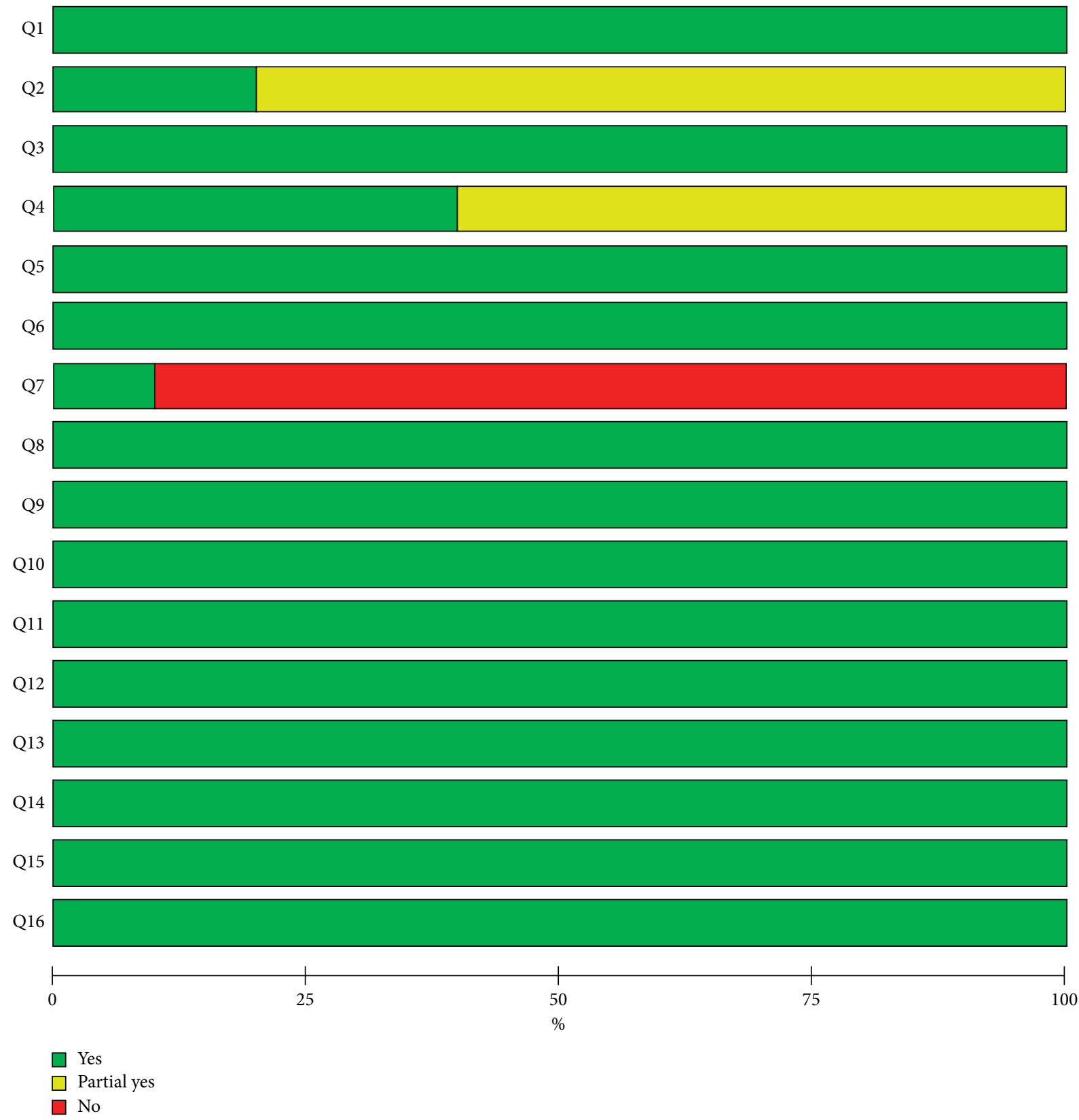

FIGURE 3: Graphical representation of the AMSTAR-2 assessments.

3.4.2. Safety of the Interventions. One study [19] reported the meta-analysis results in adverse effects, and no statistically significant difference was found between patients treated with acupuncture and CM.

\section{Discussion}

Treatment of IBS focuses on symptom management to maintain daily functioning and improve QOL. However, due to significant side effects of prescribed medications, some sufferers do not take multiple CM but instead turn to complementary and alternative therapies for remedy $[11,29]$. A number of SRs/MAs have investigated the efficacy of acupuncture for IBS patients. The purpose of this study was to systematically collate, appraise, and synthesize the evidence published in recent years.

Ten SRs/MAs regarding to the efficacy of acupuncture for IBS were finally included. From the meta-analysis results of these studies, patients reported that acupuncture had a greater benefit on IBS symptoms than CM. However, these findings must be considered cautiously, given the limitations on methodological quality, reporting quality, and evidence quality of the included studies. According to AMSTAR-2 and PRISMA checklists, most of (80\%) the included studies did not establish a protocol, which could undermine the rigor of the study and increase the risk of bias. For literature search, $60 \%$ studies only provided the search keywords but no specific search strategies, which could lead to publication 
TABle 3: Results of the PRISMA checklists.

\begin{tabular}{|c|c|c|c|c|c|c|c|c|c|c|c|c|}
\hline $\begin{array}{l}\text { Section/ } \\
\text { topic }\end{array}$ & Items & $\begin{array}{l}\text { Guo, } \\
2020\end{array}$ & $\begin{array}{l}\text { Zheng, } \\
2019\end{array}$ & $\begin{array}{l}\text { Yan, } \\
2019\end{array}$ & $\begin{array}{c}\text { Chao, } \\
2014\end{array}$ & $\begin{array}{l}\text { Manheimer, } \\
2012\end{array}$ & $\begin{array}{c}\mathrm{Fu}, \\
2018\end{array}$ & $\begin{array}{l}\text { Deng, } \\
2017\end{array}$ & $\begin{array}{c}\mathrm{Li} \\
2016\end{array}$ & $\begin{array}{l}\text { Pei, } \\
2012\end{array}$ & $\begin{array}{c}\text { Zhao, } \\
2010\end{array}$ & $\begin{array}{c}\text { Compliance } \\
(\%)\end{array}$ \\
\hline Title & Q1. Title & $\mathrm{Y}$ & $\mathrm{Y}$ & $\mathrm{Y}$ & $\mathrm{Y}$ & $\mathrm{Y}$ & $\mathrm{Y}$ & $\mathrm{Y}$ & $\mathrm{Y}$ & $\mathrm{Y}$ & $\mathrm{Y}$ & 100 \\
\hline Abstract & $\begin{array}{l}\text { Q2. Structured } \\
\text { summary }\end{array}$ & $\mathrm{Y}$ & $\mathrm{Y}$ & $\mathrm{Y}$ & $\mathrm{Y}$ & $\mathrm{Y}$ & Y & $\mathrm{Y}$ & $\mathrm{Y}$ & $\mathrm{Y}$ & $\mathrm{Y}$ & 100 \\
\hline \multirow{8}{*}{ Introduction } & Q3. Rationale & $\mathrm{Y}$ & $\mathrm{Y}$ & $\mathrm{Y}$ & $\mathrm{Y}$ & $\mathrm{Y}$ & $\mathrm{Y}$ & $\mathrm{Y}$ & $\mathrm{Y}$ & $\mathrm{Y}$ & $\mathrm{Y}$ & 100 \\
\hline & Q4. Objectives & $\mathrm{Y}$ & $\mathrm{Y}$ & $\mathrm{Y}$ & $\mathrm{Y}$ & $\mathrm{Y}$ & $\mathrm{Y}$ & $\mathrm{Y}$ & $\mathrm{Y}$ & $\mathrm{Y}$ & $\mathrm{Y}$ & 100 \\
\hline & $\begin{array}{l}\text { Q5. Protocol and } \\
\text { registration }\end{array}$ & $\mathrm{Y}$ & $\mathrm{N}$ & $\mathrm{N}$ & $\mathrm{N}$ & $\mathrm{Y}$ & $\mathrm{N}$ & $\mathrm{N}$ & $\mathrm{N}$ & $\mathrm{N}$ & $\mathrm{N}$ & 20 \\
\hline & $\begin{array}{l}\text { Q6. Eligibility } \\
\text { criteria }\end{array}$ & $\mathrm{Y}$ & $\mathrm{Y}$ & $\mathrm{Y}$ & $\mathrm{Y}$ & $\mathrm{Y}$ & $\mathrm{Y}$ & $\mathrm{Y}$ & $\mathrm{Y}$ & $\mathrm{Y}$ & $\mathrm{Y}$ & 100 \\
\hline & $\begin{array}{l}\text { Q7. Information } \\
\text { sources }\end{array}$ & $\mathrm{Y}$ & $\mathrm{Y}$ & $\mathrm{Y}$ & $\mathrm{Y}$ & $\mathrm{Y}$ & $\mathrm{Y}$ & $\mathrm{Y}$ & $\mathrm{Y}$ & $\mathrm{Y}$ & $\mathrm{Y}$ & 100 \\
\hline & Q8. Search & $\mathrm{Y}$ & $\mathrm{Y}$ & $\mathrm{Y}$ & PY & $\mathrm{Y}$ & PY & PY & PY & PY & PY & 40 \\
\hline & $\begin{array}{l}\text { Q9. Study } \\
\text { selection }\end{array}$ & $\mathrm{Y}$ & $\mathrm{Y}$ & $\mathrm{Y}$ & $\mathrm{Y}$ & $\mathrm{Y}$ & $\mathrm{Y}$ & $\mathrm{Y}$ & $\mathrm{Y}$ & $\mathrm{Y}$ & $\mathrm{Y}$ & 100 \\
\hline & $\begin{array}{c}\text { Q10. Data } \\
\text { collection process }\end{array}$ & $\mathrm{Y}$ & $\mathrm{Y}$ & $\mathrm{Y}$ & $\mathrm{Y}$ & $\mathrm{Y}$ & $\mathrm{Y}$ & $\mathrm{Y}$ & $\mathrm{Y}$ & $\mathrm{Y}$ & $\mathrm{Y}$ & 100 \\
\hline \multirow[t]{9}{*}{ Methods } & Q11. Data items & $\mathrm{Y}$ & $\mathrm{Y}$ & $\mathrm{Y}$ & $\mathrm{Y}$ & $\mathrm{Y}$ & $\mathrm{Y}$ & $\mathrm{Y}$ & $\mathrm{Y}$ & $\mathrm{Y}$ & $\mathrm{Y}$ & 100 \\
\hline & $\begin{array}{l}\text { Q12. Risk of bias } \\
\text { in individual } \\
\text { studies }\end{array}$ & $\mathrm{Y}$ & $\mathrm{Y}$ & $\mathrm{Y}$ & $\mathrm{Y}$ & $\mathrm{Y}$ & $\mathrm{Y}$ & $\mathrm{Y}$ & $\mathrm{Y}$ & $\mathrm{Y}$ & $\mathrm{Y}$ & 100 \\
\hline & $\begin{array}{l}\text { Q13. Summary } \\
\text { measures }\end{array}$ & $\mathrm{Y}$ & $\mathrm{Y}$ & $\mathrm{Y}$ & $\mathrm{Y}$ & $\mathrm{Y}$ & $\mathrm{Y}$ & $\mathrm{Y}$ & $\mathrm{Y}$ & $\mathrm{Y}$ & $\mathrm{Y}$ & 100 \\
\hline & $\begin{array}{l}\text { Q14. Synthesis of } \\
\text { results }\end{array}$ & $\mathrm{Y}$ & $\mathrm{Y}$ & $\mathrm{Y}$ & $\mathrm{Y}$ & $\mathrm{Y}$ & $\mathrm{Y}$ & $\mathrm{Y}$ & $\mathrm{Y}$ & $\mathrm{Y}$ & $\mathrm{Y}$ & 100 \\
\hline & $\begin{array}{l}\text { Q15. Risk of bias } \\
\text { across studies }\end{array}$ & $\mathrm{Y}$ & $\mathrm{Y}$ & $\mathrm{Y}$ & $\mathrm{Y}$ & $\mathrm{Y}$ & $\mathrm{Y}$ & $\mathrm{Y}$ & $\mathrm{Y}$ & $\mathrm{Y}$ & $\mathrm{Y}$ & 100 \\
\hline & $\begin{array}{l}\text { Q16. Additional } \\
\text { analyses }\end{array}$ & $\mathrm{Y}$ & $\mathrm{Y}$ & $\mathrm{Y}$ & $\mathrm{Y}$ & $\mathrm{Y}$ & $\mathrm{Y}$ & $\mathrm{Y}$ & $\mathrm{Y}$ & $\mathrm{Y}$ & $\mathrm{Y}$ & 100 \\
\hline & $\begin{array}{l}\text { Q17. Study } \\
\text { selection }\end{array}$ & $\mathrm{Y}$ & $\mathrm{Y}$ & $\mathrm{Y}$ & $\mathrm{Y}$ & $\mathrm{Y}$ & $\mathrm{Y}$ & $\mathrm{Y}$ & $\mathrm{Y}$ & $\mathrm{Y}$ & $\mathrm{Y}$ & 100 \\
\hline & $\begin{array}{l}\text { Q18. Study } \\
\text { characteristics }\end{array}$ & $\mathrm{Y}$ & $\mathrm{Y}$ & $\mathrm{Y}$ & $\mathrm{Y}$ & $\mathrm{Y}$ & $\mathrm{Y}$ & $\mathrm{Y}$ & $\mathrm{Y}$ & $\mathrm{Y}$ & $\mathrm{Y}$ & 100 \\
\hline & $\begin{array}{l}\text { Q19. Risk of bias } \\
\text { within studies }\end{array}$ & $\mathrm{Y}$ & $\mathrm{Y}$ & $\mathrm{Y}$ & $\mathrm{Y}$ & $\mathrm{Y}$ & $\mathrm{Y}$ & $\mathrm{Y}$ & $\mathrm{Y}$ & $\mathrm{Y}$ & $\mathrm{Y}$ & 100 \\
\hline \multirow[t]{5}{*}{ Results } & $\begin{array}{l}\text { Q20. Results of } \\
\text { individual studies }\end{array}$ & $\mathrm{Y}$ & $\mathrm{Y}$ & $\mathrm{Y}$ & $\mathrm{Y}$ & $\mathrm{Y}$ & $\mathrm{Y}$ & $\mathrm{Y}$ & $\mathrm{Y}$ & $\mathrm{Y}$ & $\mathrm{Y}$ & 100 \\
\hline & $\begin{array}{l}\text { Q21. Synthesis of } \\
\text { results }\end{array}$ & $\mathrm{Y}$ & $\mathrm{Y}$ & $\mathrm{Y}$ & $\mathrm{Y}$ & $\mathrm{Y}$ & $\mathrm{Y}$ & $\mathrm{Y}$ & $\mathrm{Y}$ & $\mathrm{Y}$ & $\mathrm{Y}$ & 100 \\
\hline & $\begin{array}{l}\text { Q22 Risk of bias } \\
\text { across studies }\end{array}$ & $\mathrm{Y}$ & $\mathrm{Y}$ & $\mathrm{Y}$ & $\mathrm{Y}$ & $\mathrm{Y}$ & $\mathrm{Y}$ & $\mathrm{Y}$ & $\mathrm{Y}$ & $\mathrm{Y}$ & $\mathrm{Y}$ & 100 \\
\hline & $\begin{array}{l}\text { Q23. Additional } \\
\text { analysis }\end{array}$ & $\mathrm{Y}$ & $\mathrm{Y}$ & $\mathrm{Y}$ & $\mathrm{Y}$ & $\mathrm{Y}$ & $\mathrm{Y}$ & $\mathrm{Y}$ & $\mathrm{Y}$ & $\mathrm{Y}$ & $\mathrm{Y}$ & 100 \\
\hline & $\begin{array}{l}\text { Q24. Summary of } \\
\text { evidence }\end{array}$ & $\mathrm{Y}$ & $\mathrm{Y}$ & $\mathrm{Y}$ & $\mathrm{Y}$ & $\mathrm{Y}$ & $\mathrm{Y}$ & $\mathrm{Y}$ & $\mathrm{Y}$ & $\mathrm{Y}$ & $\mathrm{Y}$ & 100 \\
\hline \multirow{2}{*}{ Discussion } & Q25. Limitations & $\mathrm{Y}$ & $\mathrm{Y}$ & $\mathrm{Y}$ & $\mathrm{Y}$ & $\mathrm{Y}$ & $\mathrm{Y}$ & $\mathrm{Y}$ & $\mathrm{Y}$ & $\mathrm{Y}$ & $\mathrm{Y}$ & 100 \\
\hline & Q26. Conclusions & $\mathrm{Y}$ & $\mathrm{Y}$ & $\mathrm{Y}$ & $\mathrm{Y}$ & $\mathrm{Y}$ & $\mathrm{Y}$ & $\mathrm{Y}$ & $\mathrm{Y}$ & $\mathrm{Y}$ & $\mathrm{Y}$ & 100 \\
\hline Funding & Q27. Funding & $\mathrm{Y}$ & $\mathrm{Y}$ & $\mathrm{Y}$ & $\mathrm{Y}$ & $\mathrm{Y}$ & $\mathrm{N}$ & $\mathrm{Y}$ & $\mathrm{Y}$ & $\mathrm{Y}$ & $\mathrm{Y}$ & 90 \\
\hline
\end{tabular}

bias and undermined the credibility of the results. Moreover, $90 \%$ studies did not provide the lists of excluded trails, which may undermine the transparency of the study process. According to the GRADE tool, no outcome indicators provided high-quality evidence, indicating that the metaanalyses results of the included studies may differ from the true results. The risk of bias for the enrolled trails of the included studies was the main reason for evidence degradation. Further analyses found common limitations of the enrolled trails as follows: only randomization was mentioned without the randomization method; the allocation was not concealed; and only single blinding was implemented. Therefore, the basic factor leading to the decline in the quality of evidence was the low methodological quality of the enrolled trails. It was believed that well-designed and implemented randomized controlled trials were considered to be the gold standard to avoid the risk of bias [30]. Furthermore, almost all of the included SRs/MAs indicated that acupuncture seemed to have a significant clinical efficacy for IBS; however, most authors did not wish to draw clear conclusions due to low methodological quality or the small size of the enrolled trails. 
TABle 4: Certainty of evidence quality.

\begin{tabular}{|c|c|c|c|c|c|c|c|c|c|}
\hline Studies & Treatments & Outcomes & Limitations & Inconsistency & Indirectness & Imprecision & $\begin{array}{c}\text { Publication } \\
\text { bias }\end{array}$ & $\begin{array}{c}\text { Relative } \\
\text { effect ( } 95 \% \\
\text { CI) } \\
\end{array}$ & Quality \\
\hline \multirow{7}{*}{ Guo et al. [19] } & \multirow{7}{*}{$\begin{array}{l}\text { AT versus } \\
\text { CM }\end{array}$} & $\begin{array}{c}\text { Weekly } \\
\text { defecation }\end{array}$ & -1 & 0 & 0 & 0 & 0 & $\begin{array}{l}\text { SMD, } \\
-0.29 \\
(-0.49 \\
-0.08)\end{array}$ & M \\
\hline & & $\begin{array}{c}\text { IBS } \\
\text { symptom } \\
\text { scores }\end{array}$ & -1 & 0 & 0 & 0 & 0 & $\begin{array}{l}\text { SMD, } \\
-1.17 \\
(-1.42, \\
-0.93)\end{array}$ & M \\
\hline & & IBS-QOL & -1 & 0 & 0 & -1 & 0 & $\begin{array}{l}\text { SMD } 2.37 \\
(1.94,2.80)\end{array}$ & $\mathrm{L}$ \\
\hline & & IBS-SSS & -1 & 0 & 0 & 0 & 0 & $\begin{array}{c}\text { SMD -0.75 } \\
\quad(-1.04 \\
\quad-0.47)\end{array}$ & M \\
\hline & & Effective rate & -1 & 0 & 0 & 0 & 0 & $\begin{array}{c}\text { RR } 1.25 \\
(1.18,1.32)\end{array}$ & M \\
\hline & & $\begin{array}{l}\text { Recurrence } \\
\text { rate }\end{array}$ & -1 & 0 & 0 & -1 & 0 & $\begin{array}{c}\text { RR } 0.43 \\
(0.28,0.66)\end{array}$ & $\mathrm{L}$ \\
\hline & & $\begin{array}{l}\text { Adverse } \\
\text { effects }\end{array}$ & -1 & 0 & 0 & -1 & 0 & $\begin{array}{c}\text { RR } 0.59 \\
(0.12,2.90)\end{array}$ & $\mathrm{L}$ \\
\hline \multirow{5}{*}{$\begin{array}{l}\text { Zheng et al. } \\
{[20]}\end{array}$} & \multirow{2}{*}{$\begin{array}{l}\text { AT versus } \\
\text { Sham AT }\end{array}$} & Effective rate & -1 & 0 & 0 & 0 & 0 & $\begin{array}{c}\text { RR } 1.22 \\
(1.01,1.47)\end{array}$ & M \\
\hline & & IBS-QOL & -1 & 0 & 0 & 0 & 0 & $\begin{array}{c}\text { SMD }-0.10 \\
(-0.31 \\
0.11)\end{array}$ & M \\
\hline & \multirow{3}{*}{$\begin{array}{l}\text { AT versus } \\
\text { CM }\end{array}$} & Effective rate & -1 & 0 & 0 & 0 & 0 & $\begin{array}{c}\text { RR } 1.17 \\
(1.12,1.23)\end{array}$ & M \\
\hline & & $\begin{array}{c}\text { IBS } \\
\text { symptom } \\
\text { scores }\end{array}$ & -1 & -1 & 0 & 0 & 0 & $\begin{array}{c}\text { SMD -1.16 } \\
\quad(-1.61 \\
-0.71)\end{array}$ & $\mathrm{L}$ \\
\hline & & IBS-QOL & -1 & 0 & 0 & -1 & 0 & $\begin{array}{l}\text { SMD } 0.75 \\
(0.34,1.16)\end{array}$ & $\mathrm{L}$ \\
\hline \multirow[b]{2}{*}{ Yan et al. [21] } & \multirow[b]{2}{*}{$\begin{array}{l}\mathrm{AT}+\mathrm{CHM} \\
\text { versus CM }\end{array}$} & Effective rate & -1 & 0 & 0 & 0 & 0 & $\begin{array}{c}\text { RR } 1.29 \\
(1.24,1.35)\end{array}$ & M \\
\hline & & $\begin{array}{l}\text { Abdominal } \\
\text { pain }\end{array}$ & -1 & -1 & 0 & 0 & 0 & $\begin{array}{c}\text { SMD }-0.45 \\
\quad(-0.72 \\
\quad-0.17)\end{array}$ & $\mathrm{L}$ \\
\hline $\begin{array}{l}\text { Chao and } \\
\text { Zhang [22] }\end{array}$ & $\begin{array}{l}\text { AT versus } \\
\text { CM }\end{array}$ & Effective rate & -1 & 0 & 0 & 0 & 0 & $\begin{array}{c}\text { RR } 1.75 \\
(1.24,2.46)\end{array}$ & M \\
\hline \multirow{3}{*}{$\begin{array}{l}\text { Manheimer } \\
\text { et al. [23] }\end{array}$} & \multirow{2}{*}{$\begin{array}{l}\text { AT versus } \\
\text { Sham AT }\end{array}$} & IBS-SSS & -1 & 0 & 0 & -1 & 0 & $\begin{array}{c}\text { SMD }-0.11 \\
(-0.35 \\
0.13)\end{array}$ & $\mathrm{L}$ \\
\hline & & IBS-QOL & -1 & 0 & 0 & -1 & 0 & $\begin{array}{c}\text { SMD }-0.03 \\
\quad(-0.27 \\
0.22)\end{array}$ & $\mathrm{L}$ \\
\hline & $\begin{array}{l}\text { AT versus } \\
\text { CM }\end{array}$ & Effective rate & -1 & 0 & 0 & 0 & 0 & $\begin{array}{c}\text { RR } 1.28 \\
(1.12,1.45)\end{array}$ & M \\
\hline $\begin{array}{l}\text { Fu and Jiang } \\
{[24]}\end{array}$ & $\begin{array}{l}\text { AT versus } \\
\text { CM }\end{array}$ & Effective rate & -1 & 0 & 0 & 0 & 0 & $\begin{array}{c}\text { RR } 1.20 \\
(1.15,1.25)\end{array}$ & M \\
\hline \multirow{2}{*}{$\begin{array}{l}\text { Deng et al. } \\
\text { [25] }\end{array}$} & \multirow{2}{*}{$\begin{array}{l}\text { AT versus } \\
\quad \mathrm{CM}\end{array}$} & Effective rate & -1 & 0 & 0 & 0 & 0 & $\begin{array}{c}\text { OR } 3.92 \\
(2.83,5.43)\end{array}$ & M \\
\hline & & $\begin{array}{c}\text { Recurrence } \\
\text { rate }\end{array}$ & -1 & 0 & 0 & -1 & 0 & $\begin{array}{c}\text { OR } 0.22 \\
(0.12,0.41)\end{array}$ & $\mathrm{L}$ \\
\hline \multirow{2}{*}{ Li et al. [26] } & \multirow{2}{*}{$\begin{array}{l}\text { AT versus } \\
\quad \mathrm{CM}\end{array}$} & $\begin{array}{l}\text { Recurrence } \\
\text { rate }\end{array}$ & -1 & 0 & 0 & -1 & -1 & $\begin{array}{c}\text { RR } 0.49 \\
(0.35,0.68)\end{array}$ & CL \\
\hline & & Effective rate & -1 & 0 & 0 & 0 & -1 & $\begin{array}{c}\text { RR } 1.17 \\
(1.08,1.26)\end{array}$ & $\mathrm{L}$ \\
\hline Pei et al. [27] & $\begin{array}{l}\text { AT versus } \\
\text { CM }\end{array}$ & Effective rate & -1 & -1 & 0 & 0 & 0 & $\begin{array}{c}\text { RR } 1.27 \\
(1.09,1.49)\end{array}$ & $\mathrm{L}$ \\
\hline Zhao [28] & $\begin{array}{c}\text { AT versus } \\
\text { CM }\end{array}$ & Effective rate & -1 & 0 & 0 & 0 & -1 & $\begin{array}{c}\text { RR } 1.28 \\
(1.20,1.38)\end{array}$ & $\mathrm{L}$ \\
\hline
\end{tabular}


The action mechanism of acupuncture for IBS includes regulating the gastrointestinal motility, reducing visceral hypersensitivity, regulating the brain-intestine axis, reducing low-level intestinal mucosal inflammation, promoting intestinal microflora balance, and adjusting psycho-psychological status [31]. IBS is a gastrointestinal disorder in which intestinal spasm causes abdominal pain, hypermotility leads to diarrhea, and hypomotility leads to constipation. Thus, for the purpose of treatment, IBS can be divided into three types: constipation-predominant, diarrhea-predominant, or mixed [32]. Animal experiments revealed that acupuncture stimulation of IBS-D model rats effectively improved diarrhea symptoms in rats, and it was found that the mRNA and protein expression of APQ8 in the rat colon tissue was reduced, while the protein expression of VIP was increased [33]. For patients with IBS-C, electroacupuncture stimulation of Zusanli can promote contraction of the patient's colon ends and accelerate colonic transit, which in turn improves constipation symptoms [34]. These results suggest that acupuncture has a bidirectional regulatory effect on intestinal motility in IBS patients. Furthermore, EA intervention can ameliorate the fecal property in IBS-C rats, which may be associated with its function in inhibiting the expression of colonic CGRP and SP proteins [35]. Visceral hypersensitivity is considered an important pathological mechanism in the development of IBS. It is reported that EA can alleviate visceral hypersensitivity in IBS-D and IBS-C rats by regulating the expression level of TRPV1 in the colon $[35,36]$. The brain-gut axis was a complex, bidirectional signaling system between the central nervous system and the gastrointestinal system. It is reported that acupuncture could improve intestinal motility and visceral sensitivity by modulating brain-gut peptide levels in the central nervous system, gut, and blood [31]. Furthermore, electroacupuncture decreases 5-HT and CGRP, increases NPY in the brain-gut axis in rat models of IBS-D [37], and increases the number of neurons in the myenteric plexus of IBS-C rats [38]. Posttraumatic stress disorder (PTSD) is thought to be associated with IBS and is a common comorbidity [39]. It is reported that acupuncture can affect the autonomic nervous system, and the prefrontal as well as limbic brain structures, enabling it to relieve the symptoms of PTSD [40]. Activation of the immune system was strongly associated with IBS, and acupuncture could downregulate the expression of serum IL-18, TNF- $\alpha$, and IL23 in IBS patients, thus playing an immunoregulatory role [41]. The overgrowth of intestinal flora may be an important factor in the induction of IBS [42]. It is reported that acupuncture treatment may modulate intestinal bacteria and the psychological state tends to balance to relieve the symptoms of IBS [31, 43]. However, there is still a lack of evidence on the regulation of intestinal microbiota in IBS through the use of acupuncture.

This overview would provide some useful information on unique treatments in clinical practice for physicians in the management of IBS, thus providing more treatment options for IBS patients. However, we found that the majority of the included reviews were of poor quality, which could result in them having low credibility. Furthermore, the
AMSTAR-2 tool, PRISMA checklist, and the GRADE system are highly subjective. Thus, different reviewers may have their own independent judgments on the evaluation results. Even with two independent reviewers in this study, subjective factors or errors cannot be completely eliminated. Finally, there is limited evidence for the efficacy of acupuncture for IBS subtypes, especially IBS-C. Further clinical and mechanistic studies of acupuncture for IBS subtypes are still necessary.

\section{Conclusion}

Acupuncture may be a promising treatment for IBS, and it could be used as an adjunct in clinical settings to improve efficacy. However, this conclusion must be treated with caution since the quality of SRs/MAs providing evidence is generally low.
Abbreviations
IBS: Irritable bowel syndrome
SR: $\quad$ Systematic review
MA: Meta-analysis
AMSTAR- Assessment of Multiple Systematic Reviews 2
2:
PRISMA: Preferred Reporting Item for Systematic
Reviews and Meta-Analyses
GRADE: Grading of Recommendations, Assessment, Development, and Evaluation
QOL: Quality of life
SSS: $\quad$ Symptom Severity Scale
CM: $\quad$ Conventional medication.

\section{Data Availability}

All analyses were based on previously published studies.

\section{Consent}

No informed consent was required.

\section{Disclosure}

Jinke Huang and Mengxiong Lu are the co-first authors.

\section{Conflicts of Interest}

The authors declare that there are no conflicts of interest.

\section{Authors' Contributions}

Jinke Huang and Mengxiong Lu initiated the study design and drafted the manuscript. Both these authors have contributed equally to this work. Jinxin Ma, Jing Ma, Xiangxue Ma, Yitian Wang, Yijun Zheng, and Kunli Zhang helped with implementation to this work. All authors read and approved the final manuscript. Fengyun Wang and Xudong Tang contributed to the methodology, review, and editing of the manuscript. All authors read and approved the final manuscript. 


\section{Acknowledgments}

This study was supported by the National Natural Science Foundation of China (nos. 81830118, 81804089, 81774303, and 82174363), State Administration of Traditional Chinese Medicine Digestive Refractory Disease Inheritance and Innovation Team Project (no. 04-212), Major Guiding Project of the China Academy of Chinese Medical Sciences (no. ZZ13-ZD-02), and the Special Fund for Basic Scientific Research Business of Central Public Welfare Scientific Research Institute (no. ZZ13-YQ-003).

\section{References}

[1] B. E. Lacy, M. Pimentel, D. M. Brenner et al., "ACG clinical guideline: management of irritable bowel syndrome," American Journal of Gastroenterology, vol. 116, no. 1, pp. 17-44, 2021.

[2] P. Moayyedi, C. N. Andrews, G. MacQueen et al., "Canadian association of gastroenterology clinical practice guideline for the management of irritable bowel syndrome (IBS)," Journal of the Canadian Association of Gastroenterology, vol. 2, no. 1, pp. 6-29, 2019.

[3] A. D. Sperber, D. Dumitrascu, S. Fukudo et al., "The global prevalence of IBS in adults remains elusive due to the heterogeneity of studies: a Rome foundation working team literature review," Gut, vol. 66, no. 6, pp. 1075-1082, 2017.

[4] Y. Long, Z. Huang, Y. Deng et al., "Prevalence and risk factors for functional bowel disorders in south China: a population based study using the Rome III criteria," Neuro-Gastroenterology and Motility: The Official Journal of the European Gastrointestinal Motility Society, vol. 29, no. 1, pp. 1-9, 2017.

[5] Y. Ringel, R. E. Williams, L. Kalilani, and S. F. Cook, "Prevalence, characteristics, and impact of bloating symptoms in patients with irritable bowel syndrome," Clinical Gastroenterology and Hepatology, vol. 7, no. 1, pp. 68-72, 2009.

[6] D. A. Drossman, C. B. Morris, S. Schneck et al., "International survey of patients with IBS: symptom features and their severity, health status, treatments, and risk taking to achieve clinical benefit," Journal of Clinical Gastroenterology, vol. 43, no. 6, pp. 541-550, 2009.

[7] S. Maxion-Bergemann, F. Thielecke, F. Abel, and R. Bergemann, "Costs of irritable bowel syndrome in the UK and US," PharmacoEconomics, vol. 24, no. 1, pp. 21-37, 2006.

[8] A. C. Ford and N. J. Talley, "IBS in 2010: advances in pathophysiology, diagnosis and treatment," Nature Reviews Gastroenterology \& Hepatology, vol. 8, no. 2, pp. 76-78, 2011.

[9] M. Corsetti and P. Whorwell, "Novel pharmacological therapies for irritable bowel syndrome," Expert Review of Gastroenterology \& Hepatology, vol. 10, no. 7, pp. 807-815, 2016.

[10] Q. X. Ng, A. Y. S. Soh, W. Loke, D. Y. Lim, and W.-S. Yeo, "The role of inflammation in irritable bowel syndrome (IBS)," Journal of Inflammation Research, vol. 11, pp. 345-349, 2018.

[11] E. M. M. Quigley, M. Fried, K.-A. Gwee et al., "World gastroenterology organisation global guidelines irritable bowel syndrome," Journal of Clinical Gastroenterology, vol. 50, no. 9, pp. 704-713, 2016.

[12] J. Huang, M. Wu, S. Liang et al., "A critical overview of systematic reviews and meta-analyses on acupuncture for poststroke insomnia," Evidence-based Complementary and Alternative Medicine, vol. 2020, Article ID 2032575, 7 pages, 2020 .
[13] K. I. Bougioukas, E. Vounzoulaki, C. D. Mantsiou et al., "Methods for depicting overlap in overviews of systematic reviews: an introduction to static tabular and graphical displays," Journal of Clinical Epidemiology, vol. 132, pp. 34-45, 2021.

[14] H. Hunt, A. Pollock, P. Campbell, L. Estcourt, and G. Brunton, "An introduction to overviews of reviews: planning a relevant research question and objective for an overview," Systematic Reviews, vol. 7, no. 1, p. 39, 2018.

[15] J. N. Lavis, "How can we support the use of systematic reviews in policymaking?" PLoS Medicine, vol. 6, no. 11, Article ID e1000141, 2009.

[16] A. Liberati, D. G. Altman, J. Tetzlaff et al., “The PRISMA statement for reporting systematic reviews and meta-analyses of studies that evaluate healthcare interventions: explanation and elaboration," BMJ, vol. 339, Article ID b2700, 2009.

[17] B. J. Shea, B. C. Reeves, G. Wells et al., "AMSTAR 2: a critical appraisal tool for systematic reviews that include randomised or non-randomised studies of healthcare interventions, or both," BMJ, vol. 358, Article ID j4008, 2017.

[18] D. Atkins, M. Eccles, S. Flottorp et al., "Systems for grading the quality of evidence and the strength of recommendations I: critical appraisal of existing approaches the GRADE working group," BMC Health Services Research, vol. 4, p. 38, 2004.

[19] J. Guo, X. Xing, J. Wu et al., "Acupuncture for adults with diarrhea-predominant irritable bowel syndrome or functional diarrhea: a systematic review and meta-analysis," Neural Plasticity, vol. 2020, Article ID 8892184, 16 pages, 2020.

[20] H. Zheng, R. Chen, X. Zhao et al., "Comparison between the effects of acupuncture relative to other controls on irritable bowel syndrome: a meta-analysis," Pain Research \& Management, vol. 2019, Article ID 2871505, 13 pages, 2019.

[21] J. Yan, Z. W. Miao, J. Lu et al., “Acupuncture plus Chinese herbal medicine for irritable bowel syndrome with diarrhea: a systematic review and meta-analysis," Evidence-Based Complementary and Alternative Medicine, vol. 2019, Article ID 7680963, 16 pages, 2019.

[22] G.-Q. Chao and S. Zhang, "Effectiveness of acupuncture to treat irritable bowel syndrome: a meta-analysis," World Journal of Gastroenterology, vol. 20, no. 7, pp. 1871-1877, 2014.

[23] E. Manheimer, K. Cheng, L. S. Wieland et al., "Acupuncture for treatment of irritable bowel syndrome," Cochrane Database of Systematic Reviews, vol. 5, no. 5, Article ID CD005111, 2012.

[24] C. W. Fu and W. Jiang, "The effect of acupuncture therapy on irritable bowel syndrome: a meta-analysis," Asia-Pacific Traditional Medicine, vol. 14, no. 12, pp. 101-105, 2018.

[25] D. X. Deng, K. K. Guo, J. Tan et al., "Acupuncture for diarrhea-predominant irritable bowel syndrome: a meta-analysis," Chinese Acupuncture and Moxibustion, vol. 37, no. 8, pp. 907-912, 2017.

[26] L. Ruigen, W. Wei, X. Ri, L. Bo, B. Xiaobei, and B. Weijing, "Meta analysis of acupuncture in treatment of irritable bowel syndrome," Global Traditional Chinese Medicine, vol. 9, no. 6, pp. 772-776, 2016.

[27] L. X. Pei, X. C. Zhang, J. H. Sun, H. Geng, and X. Wu, "Meta analysis of acupuncture-moxibustion in treatment of irritable bowel syndrome," Chinese Acupuncture and Moxibustion, vol. 32, no. 10, pp. 957-960, 2012.

[28] C. Zhao, J. P. Mu, Y. H. Cui, L. Yang, X. Ma, and L. Qi, "Metaanalysis on acupuncture and moxibustion for irritable bowel syndrome," Chinese Archives of Traditional Chinese Medicine, vol. 28, no. 5, pp. 961-963, 2010. 
[29] Y.-K. Dai, Y.-B. Wu, R.-L. Li et al., "Efficacy and safety of nonpharmacological interventions for irritable bowel syndrome in adults," World Journal of Gastroenterology, vol. 26, no. 41, pp. 6488-6509, 2020.

[30] D. Moher, S. Hopewell, K. F. Schulz et al., "CONSORT 2010 explanation and elaboration: updated guidelines for reporting parallel group randomized trials," $B M J$, vol. 10, no. 1, pp. 28-55, 2010.

[31] X.-P. Ma, J. Hong, C. P. An et al., "Acupuncture-moxibustion in treating irritable bowel syndrome: how does it work?" World Journal of Gastroenterology, vol. 20, no. 20, pp. 6044-6054, 2014.

[32] S. J. Cole, H. D. Duncan, A. H. Claydon, D. Austin, T. E. Bowling, and D. B. A. Silk, "Distal colonic motor activity in four subgroups of patients with irritable bowel syndrome," Digestive Diseases and Sciences, vol. 47, no. 2, pp. 345-355, 2002.

[33] H. H. Liu, X. D. Liu, Y. J. Wang et al., "Effects of acupoint area and non-acupoint area of eye-acupuncture on expressions of VIP and AQP8 in colonic tissues in rats with D-IBS," Chinese Acupuncture and Moxibustion, vol. 32, no. 10, pp. 919-924, 2012.

[34] S. F. Peng, T. Shi, Z. H. Shi et al., "Effect of electroacupuncture on constipation symptoms, psychological status and colonic transit function in patients with slow transit constipation," Chinese Journal of Gastroenterology and Hepatology, vol. 22, no. 3, pp. 250-253, 2013.

[35] Y. Chen, Y. Zhao, L. Wang, J.-P. Yao, Y. Li, and S.-Y. Zhou, "Involvement of Pirt/TRPV1 signaling in acupuncture-induced reduction of visceral hypersensitivity in diarrheapredominant irritable bowel syndrome rats," Zhen Ci Yan Jiu, vol. 46, no. 4, pp. 278-283, 2021.

[36] Y. N. Zhang, S. J. Wang, Y. Lu, B. B. Han, Y. Y. Zhang, and F. Z. Gai, "Impacts of electroacupuncture on the expression of TRPV1 mRNA in the rats with colon of constipation-predominant irritable bowel syndrome," World Journal of Integrated Traditional and Western Medicine, vol. 8, no. 8, pp. 778-780, 2013.

[37] J. Sun, X. Wu, Y. Meng et al., "Electro-acupuncture decreases 5-HT, CGRP and increases NPY in the brain-gut axis in two rat models of diarrhea-predominant irritable bowel syndrome (D-IBS)," BMC Complementary and Alternative Medicine, vol. 15 , no. 1 , p. $340,2015$.

[38] H.-G. Wu, B. Jiang, E.-H. Zhou et al., "Regulatory mechanism of electroacupuncture in irritable bowel syndrome: preventing MC activation and decreasing SP VIP secretion," Digestive Diseases and Sciences, vol. 53, no. 6, pp. 1644-1651, 2008.

[39] Q. X. Ng, A. Y. S. Soh, W. Loke, N. Venkatanarayanan, D. Y. Lim, and W.-S. Yeo, "Systematic review with metaanalysis: the association between post-traumatic stress disorder and irritable bowel syndrome," Journal of Gastroenterology and Hepatology, vol. 34, no. 1, pp. 68-73, 2019.

[40] H. Wahbeh, A. Senders, R. Neuendorf, and J. Cayton, "Complementary and alternative medicine for posttraumatic stress disorder symptoms: a systematic review," Journal of Evidence-Based Complementary \& Alternative Medicine, vol. 19, no. 3, pp. 161-175, 2014.

[41] R. H. Zhang, "Therapeutic efficacy of acupuncture-moxibustion for elderly irritable bowel syndrome and its effect on serum IL-18, IL-23, and TNF- $\alpha$," Chinese Journal of Gerontology, vol. 33, pp. 1435-1436, 2013.

[42] E. M. M. Quigley and O. F. Craig, "Irritable bowel syndrome; update on pathophysiology and management," Turkish Journal of Gastroenterology, vol. 23, no. 4, pp. 313-322, 2012.
[43] W. Xiaoli, H. Guangrui, L. Xiaolu et al., "Research progress on acupuncture and moxibustion regulating intestinal bacteria," Acta Universitatis Traditionis Medicalis Sinensis Pharmacologiaeque Shanghai, vol. 35, no. 2, pp. 103-108, 2021. 